Original Article

www.pjkd.com.pk

\title{
Preeclampsia: Its Contribution to Pregnancy Related Acute Kidney Injury. A Tertiary Care Nephrology Unit Experience
}

\author{
Rubina Naqvi \\ Sindh Institute of Urology and Transplantation (SIUT), Karachi. Pakistan
}

Abstract:

Introduction: Acute kidney injury (AKI) is a commonly recognized clinical problem after many morbid conditions related to complicated obstetrics. Preeclampsia, eclampsia and hemolysis, elevated liver enzymes and thrombocytopenia (HELLP) are among many other conditions of pregnancy related AKI (PR-AKI). Pathogenesis of preeclampsia is complex and not clearly understood though many researchers have published numerable studies on subject.

Present study aims to report case series of patients with PR-AKI developing in association with preeclampsia, their course of illness and outcome.

Patients and Methods: This study was carried out at Sindh Institute of Urology and Transplantation, Karachi, Pakistan, which is a tertiary care renal unit. Subjects for the study reported here comprise a cohort of 227 women coming to this institution with diagnosis of preeclampsia and AKI. AKI was defined according to KDIGO guidelines and diagnosis of preeclampsia was based on latest definition from UpToDate.

Results: From January 1990 to December 2014, there were 1,441 women with PR-AKI brought to this institution among these 227 (15.75\%) fulfilled the criteria of preeclampsia. Mean age of these women was $27.96 \pm 5.54$ years. On presentation 93.4\% were oligo-anuric, thrombocytopenia was found in 48\%, deranged INR in 18.9\% and raised liver enzymes in 33.5\%. On arrival 22\% were in sepsis, mechanical ventilator support required in $11 \%$. Impaired Glasgow coma scale noticed in $22 \%$. Intra uterine death of fetus was reported in $75 \%$. Antepartum hemorrhage in 38\% and post partum hemorrhage in $32 \%$. More than one insults combination was found in many. Renal replacement therapy in form of hemodialysis was conducted in $94.7 \%$. Complete recovery seen in 25\%, partial recovery in 31\%, CKD developed in 4.4\%, ESKD in $27.3 \%$ and $12 \%$ died during acute phase of illness. Partial recovery labelled in those who were showing improving trends in renal function but lost follow up before 90 days of insult. While CKD were women who remained in long term follow up with abnormal renal function and not requiring renal replacement. Sepsis, multi-organ failure and requirement of mechanical ventilatory support on arrival were significant predictors of mortality with $\mathrm{p}$ value $<0.001$.

Conclusion: Preeclampsia and its progress to eclampsia can be severe life-threatening conditions especially when patients present with sepsis and multi organ failure. Many women develop irreversible AKI in these conditions.

Key Words: Acute Kidney Injury (AKI), Pregnancy related AKI (PR-AKI), Preeclampsia, Eclampsia, HELLP syndrome, Pakistan.

\section{Corresponding Author}

Dr Rubina Naqvi, MBBS, MD (Nephrology), Fellow ISN, PGD Bioethics

Professor Department of Nephrology

Sindh Institute of Urology and Transplantation (SIUT)

Karachi. 74200. Pakistan

Email: rubinanaqvi@gmail.com, naqvirubina@yahoo.com

Received: February 4, 2021. Accepted March 8, 2021.

PJKD 2020;5(2):40-47

\section{Introduction:}

Preeclampsia which remains as one of great mystery of obstetrics is a syndrome characterized by hypertension, swelling and proteinuria. It affects 2-8 \% of pregnancies worldwide ${ }^{1}$, usually occurs in third trimester or late second trimester and causes substantial perinatal morbidity and mortality. It is 


\section{AKI \& Preeclampsia}

more common in first pregnancy or elderly primigravida but can occur in multiparous women as well. Preeclampsia only occurs in presence of placenta, even if there is no fetus as in cases of hydatidiform mole and spontaneously resolves in post partum period. The pathophysiology of preeclampsia has been searched over past 6-7 decades and various theories has been described. The clinical findings of preeclampsia are supportive for systemic endothelial dysfunction and microangiopathy. The target organs can be kidneys where glomerular endotheliosis occurs causing proteinuria and hypertension, liver involvement resulting in elevated liver enzymes, hemolysis and thrombocytopenia (HELLP syndrome). Brain involvement can cause headaches, blurring of vision and seizures when progress towards eclampsia. It is believed that placenta has abnormal vascular development in women who develop preeclampsia. Genetic predisposition has also been reported with positive family history. ${ }^{2}$ Prostacyclin production is reportedly decreased in preeclampsia ${ }^{3}$, whereas thromboxane increases significantly. ${ }^{4}$ The release of endothelin from vascular endothelial cells has been postulated to cause hypertension in preeclampsia. ${ }^{5}$

More recently placental secretion of anti angiogenic factors like soluble fms-like tyrosine kinase- 1 has been reported. It binds vascular endothelial growth factor in maternal circulation and causes widespread maternal vascular dysfunction leading to preeclampsia. ${ }^{6}$ It has also been reported that women with a history of recovered AKI are at 3-5 fold increased risk for preeclampsia and fetal growth retardation despite otherwise normal GFR prior to pregnancy.

Despite improvement in understanding of pathophysiology over these decades, management of preeclampsia mainly remains supportive with close observation, use of anti hypertensives, magnesium sulphate and early induction of labor. Fetal outcome is poor; fetus often dies in utero or if survives fetus is frequently small for gestational age.

The current study is aimed to report preeclampsia contributing to pregnancy related acute kidney injury (PR-AKI) from tertiary care renal unit where these patients were referred from obstetrical facilities after being found derangement in renal functions and/or decline in urine output.

\section{Patients and Methods:}

It was a retrospective, observational study involving data collection from case records, carried out at a tertiary nephrology care center of Sindh Institute of Urology and Transplantation (SIUT) over a period of 25 years, i.e. from January 1990 to December 2014. AKI was defined according to the KDIGO guidelines (criteria applied in retrospect) ${ }^{8}$ increase in serum creatinine or decline in urine output were used to classify patients as having AKI. Pregnancy related complications contribute to sizable population of AKI. Among these women only those with preeclampsia were selected for present study.

Preeclampsia was defined according to latest updated definition, as the new onset of hypertension and proteinuria or the new onset of hypertension and significant end-organ dysfunction with or without proteinuria after 20 weeks of gestation or postpartum in a previously normotensive woman.

There may found co existing HELLP syndrome (Hemolysis, Elevated Liver enzymes, Low Platelets) or hemolytic-uremic syndrome (HUS) with preeclampsia. But women with HELLP or HUS with out hypertension and edema developing after 20 weeks of gestation are not included in current study. 
Women were labelled recovered when achieved normal renal function and sustained so in long term follow up. Partial recovery was labelled in those who were showing improving trends in renal function but lost follow up before 90 days of insult. While CKD were women who remained in long term follow up with abnormal renal function and not requiring renal replacement. End stage kidney disease (ESKD) was defined when patient became dialysis dependent.

The study protocol was in accordance with the Declaration of Helsinki and SIUTs' Institutional Ethical Review Committee has granted permission for publishing this data.

Statistical Analysis: Statistical analysis was done on SPSS version 22.0. Continuous variables were expressed as mean \pm standard deviation, median and minimum and maximum ranges are documented. Frequencies and percentages were computed for categorical variables. Chi- square independent or Fisher' $\mathrm{s}$ Exact test were used to determine the proportions difference between outcomes. $\mathrm{P}$ value $\leq 0.05$ was considered as significant.

\section{Results:}

During study period between January 1990 to December 2014, there were found 1,441 cases of PR-AKI which was $25.62 \%$ of total AKI brought to this institution. Of these 227 (15.8\%) were found to have preeclampsia including HELLP syndrome or HUS in association with preeclampsia. Mean age of these women was 27.96 \pm 5.54 years (range 18-42 years). Demographics of women from day of hospitalization are given in Table 1. On presentation 212 (93.4\%) were oligo-anuric, 215 (94.7\%) required renal replacement therapy on arrival, hyponatremia was found in 69 (30.4\%), hyperkalemia in 94 (41.4\%), thrombocytopenia in 109 (48\%), thrombocytosis in 25 (11\%), deranged INR in 43(18.9\%), acidosis in $190(83.7 \%)$, raised bilirubin in $47(20.7 \%)$, raised liver enzymes in $76(33.5 \%)$, on arrival there were $50(22.02 \%)$ women in sepsis, mechanical ventilator support required in $25(11.01 \%)$. Fifty-two (22.90\%) women were found in pulmonary edema on arrival. Seizures were reported in 51 (22.46\%)

Table 1: Demographics of studied population

\begin{tabular}{|c|c|c|c|}
\hline Parameters & Mean \pm SD & Median & Range \\
\hline Age (years) & $27.960 \pm 5.544$ & 28 & $18-42$ \\
\hline Duration of Insult (days) & $8.22 \pm 6.160$ & 6 & $1-28$ \\
\hline Hemoglobin(G/dl) & $8.008 \pm 1.884$ & 7.9 & 4.1-13.4 \\
\hline$W C C \times 10^{3} / \mu l$ & $19.669 \pm 9.201$ & 17.7 & $3.7-54.2$ \\
\hline Platelet $\times 10^{3} / \mu \mathrm{l}$ & $217.05 \pm 183.701$ & 155 & $11-1189$ \\
\hline Urea mg/dl & $196.16 \pm 93.779$ & 180 & $56-547$ \\
\hline Creatinine $\mathrm{mg} / \mathrm{dl}$ & $9.672 \pm 4.907$ & 8.6 & 2.4-26.29 \\
\hline Sodium $\mathrm{mEq} / \mathrm{L}$ & $133.06 \pm 10.022$ & 135 & $100-161$ \\
\hline Potassium $\mathrm{mEq} / \mathrm{L}$ & $5.047 \pm 1.205$ & 4.5 & $2.1-8.3$ \\
\hline Venous Bicarb $\mathrm{mEq} / \mathrm{L}$ & $15.47 \pm 5.427$ & 15 & $4-42$ \\
\hline LDH U/L* & $1780.27 \pm 1641.161$ & 2345 & $112-9566$ \\
\hline Uric Acid mg/dl* & $11.033 \pm 2.376$ & 10.9 & $6.5-23.8$ \\
\hline AST U/L* & $241.67 \pm 642.233$ & 46.50 & $5-4672$ \\
\hline ALT U/L* & $169.20 \pm 361.916$ & 37 & $5-2542$ \\
\hline Alk Phos U/L* & $156.70 \pm 111.601$ & 127 & $19-1058$ \\
\hline
\end{tabular}

*not available for all patients 
women. Three women had hemiparesis developed during this acute course of illness. Along with preeclampsia other factors like perinatal hemorrhage, intra uterine death of fetus, requirement of LSCS were present with different frequencies and described in Table 2. Renal biopsies are available in 48 women in present study, in 30 it was reported patchy or extensive cortical necrosis, acute tubular necrosis in 11, tubulo interstitial in 2 and HUS in 5. Urinalysis was available for 116(51.1\%) women and all had 3-4+ proteinuria on dipstick. Many had absolute anuria on presentation and thus urinalysis not available from day of hospitalization. Outcome of all studied population is given in Fig 1.

Statistically significant impact on outcome in term of death was seen with combination of sepsis, multiorgan failure and requirement of mechanical ventilation $(\mathrm{p}<0.001)$ and sepsis and mechanical ventilation p 0.003 .

Women developing ESKD revealed highly significant association with sepsis $(\mathrm{p}<0.001)$. HELLP in association with preeclampsia was significantly higher in women who died (p 0.009).

Table 2: Associated factors with Preeclampsia

\begin{tabular}{|l|l|l|}
\hline Factors & Number & \% \\
\hline Ante Partum Hemorrhage & 86 & 37.88 \\
\hline Post Partum Hemorrhage & 73 & 32.15 \\
\hline Intra Uterine Fetal Death & 171 & 75.30 \\
\hline LSCS & 96 & 42.29 \\
\hline HELLP syndrome & 32 & 14.09 \\
\hline HUS & 5 & 2.20 \\
\hline Eclampsia & 51 & 22.46 \\
\hline *more than one factor in one woman was frequently found
\end{tabular}

\section{Discussion:}

Preeclampsia contributing to PR-AKI has been reported $28.57 \%$ from India. ${ }^{10}$ From developed world PR-AKI seen on rise over last two decade and hypertensive disorders including preeclampsia has shown statistically significant increase during 2009-2010. ${ }^{11,12}$

A study published from tertiary renal care center of same city of Karachi, addressing PR-AKI has shown preeclampsia, eclampsia and HELLP contributing $11.6 \%$ of this population, this was one year study on 43 women, overall poor outcome has been reported in this study but details of 5 women with eclampsia, preeclampsia and HELLP was not specified. ${ }^{13}$

Another study from country reveals similar frequency of $11.7 \%$ of preeclampsia, eclampsia and HELLP to total PR-AKI during one year, but again total population was small and isolated outcome from this special sub-group is not mentioned. ${ }^{14}$ Present study reveals preeclampsia, eclampsia and HELLP contributing $15.75 \%$ of total PR-AKI and highlights details of presentation and outcome in this particular group, also it is larger size population studied over a span of 25 years.

Seizures has been reported in 51 (22.46\%) women in present cohort, but it can not be commented with confidence that this was eclampsia or other metabolic factors that were contributing to seizures for example hyponatremia in 30\% with serum sodium level as low as $100 \mathrm{mEq} / \mathrm{L}$ in some and hypernatremia in $6.6 \%$ with sodium levels as high as $161 \mathrm{mEq} / \mathrm{L}$ in some women, many women were acidotic on presentation, also there were many who presented in septicemia which can contribute to seizures. 
HELLP syndrome develops in 10-20\% of women with preeclampsia. ${ }^{2}$ In present study 32 (14.09\%) women had associated HELLP.

\section{Fig 1: Outcome of preeclampsia related AKI among 227 pregnant females.}

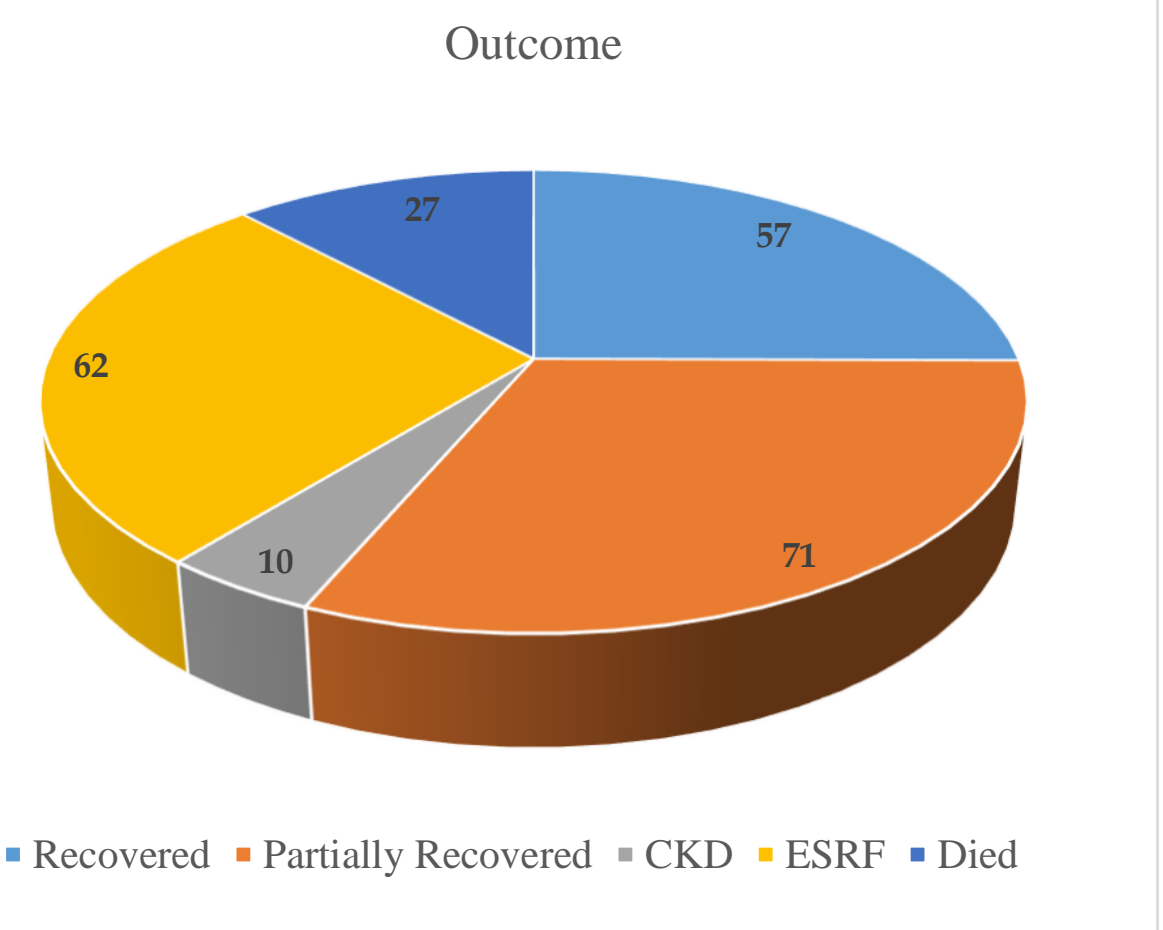

AKI in setting of preeclampsia and associated HELLP syndrome has shown other associated complications like abruptio placentae in these women. Some studies have reported abruption of placenta and antepartum or post partum hemorrhage in $22-24 \%$ of women. ${ }^{15}$ In present study antepartum hemorrhage was reported in $38 \%$ and post partum in $32 \%$ women.

Micro angiopathic hemolysis is reported to be associated with severe form of preeclampsia, ${ }^{9}$ in present series histological findings of microangiopathy has been found in 5 women. Those who had HUS with no supporting diagnosis of preeclampsia have been published from this institution previously. ${ }^{16}$

The association of hyperuricemia with pre eclampsia was first described more than a century ago ${ }^{17}$, and serum uric acid levels are usually found elevated in preeclampsia. In present study uric acid levels are not available for all patients but where done it was found raised in 98\%. Uric acid may be result of tissue ischemia and hyperuricemia may contribute to vascular damage and hypertension. ${ }^{18}$

While some have reported low absolute risk of ESKD development, ${ }^{19}$ some mentioned 21.2\% of HELLP and AKI women requiring long term dialysis ${ }^{20}$ in our experience we have found $27.3 \%$ of women developing ESKD. Urinary excretion of viable podocytes or podocyte specific tryptic peptides or their respective mRNAs has been reported and labelled as highly sensitive and specific markers for preeclampsia. ${ }^{21}$ This can explain permanent glomerular damage and irreversible AKI in this particular 


\section{AKI \& Preeclampsia}

group, but these markers were not studied in present population.

Higher risk of maternal mortality has been reported with isolated HELLP syndrome ${ }^{22}$ and this risk further increases with development of complication like AKI. In our studied population 11.9\% women died during acute phase of illness and there was significant correlation of HELLP syndrome was found with death of women in present study. In a local study by Khattak et.al. hypertensive disorders were observed in $6.8 \%$ of pregnancy related AKI compared to $15.8 \%$ in the current study ${ }^{23}$ They had far lesser number of patients $(n=88)$ compared to 1441 pregnant women with AKI in our study.

Risks for fetus also increases with preeclampsia and preterm child birth whether spontaneous or induced because of uncontrolled hypertension, small for gestational age fetuses and intra uterine fetal demise all has shown association with preeclampsia. In present study intra uterine death was reported in $75 \%$ of women indicating severity of condition before recognition.

Preterm babies also are at risk of developing neurological deficits ${ }^{25}$ we have not recorded this aspect in our present study.

Pulmonary edema has been reported as a feature of the severe preeclampsia and was observed in approximately $10 \%$ of preeclampsia with severe features in a prospective study. ${ }^{26}$ In present studied population we have also seen this feature but with little higher frequency that may explain delay in diagnosis of preeclampsia and severity of disease. As discussed previously catchment area for our renal care unit is very extensive and because of availability of free medical care and renal replacement therapy at this center very poor patients from very distant areas of country come to this institution. ${ }^{27}$ Patient' $\mathrm{S}$ relatives often stay on road side tents as long as their patients being treated here. Once they discharged from hospital till the length of few follow ups they stay there and when their patient is stable they go back to home town and never come back. That social factor is reflected in remarkable number of women in present study that is $31 \%$ labelled as partially recovered.

Limitation of study, as it was retrospective data collection from records many women from very early part of study were lacking reports available for serum uric acid, though over later years it was usually suggested for PR-AKI patients.

\section{Conclusion:}

There has been increasing interest and research going on regarding preeclampsia and other disorders causing complications during pregnancy or in post partum period. In our country with poor health infra structure even most preventable causes like simple perinatal hemorrhages causing hypovolemia and ischemic tubular injury can not be addressed appropriately. Other complications like preeclampsia, eclampsia and HELLP syndromes which have complex pathophysiology would come further down the list. To know the exact size of problem we need to do properly designed prospective control studies with implementation of further refined studies to assess podocyte excretion, long term follow up on born babies in these women etc.

Acknowledgement: Valuable help of Mr. Iqbal Mujtaba (Research Dept. SIUT) for statistical analysis of data is gratefully acknowledged. 


\section{AKI \& Preeclampsia}

\section{Conflict of Interest: None. Funding: None.}

\section{References:}

1. Duley L. The global impact of pre-eclampsia and eclampsia. Semin Perinatol. 2009; 33: 130 - 7

2. Karumanchi SA, Maynard SE, Stillman IE, Epstein FH, Sukhatme VP. Preeclamsia: A renal perspective. Kidney Int.2005;67:2101-13.

3. Fitzgerald DJ, Entman SS, Mulloy K, Fitzgerald GA. Decreased prostacyclin biosynthesis preceding the clinical manifestation of pregnancy induced hypertension. Circul.1987;75:956-63

4. Walsh SW. Preeclampsia: an imbalance in placental prostacyclin and thromboxane production. Am J Obst Gynaecol. 1985; 152(3):335-40.

5. Clark BA, Halvorson L, Sachs B, Epstein FH. Plasma endothelin levels in preeclampsia: Elevation and correlation with uric acid levels and renal impairment. Am J Obst Gynaecol. 1992;166:962-8.

6. Maynard SE, Karumanchi SA, Angiogenic factors and preeclampsia. Semin Nephrol. 2011;31(1):33

7. Tangren JS, Thadani R. Animal Model of Pregnancy after AKI Mirrors the Human Observation. JASN. 2021; 32:259-60

8. KDIGO: Definition and classification of AKI. KI Suppl. 2012;2:19-36

9. UpToDate. Topic 6814 Version 138.0. August P, Sibai BA. Preeclampsia: Clinical Features and diagnosis. Last updated Jan21, 2021.https://www.uptodate.com/contents/preeclampsia-clinical-features-and-diagnosis. Accessed 30 jan' 2021 at $1030 \mathrm{hrs}$.

10. Goplani KR, Shah PR, Gera DN, Gumber M, Dabhi M, Feroz A, Kanodia K, Suresh S, VanikarAV, Trivedi HL. Pregnancy related acute renal failure: A single center experience. Ind J Nephrol. 2008;18(1):17-21.

11. Mehrabadi A, Liu S, Bartholomew S, Hutcheon JA, Magee LA, Kramer MS, Liston RM. Hypertensive disorders of pregnancy and the recent increase in obstetric acute renal failure in Canada: population based retrospective cohort study. BMJ 2014;349:g4731 doi: 10.1136/bmj.g4731

12. Fakhouri F, Deltombe C. Pregnancy-related acute kidney injury in high income countries: Still a critical issue. J. Nephrol. 2017; 30(6):767 - 71)

13. Hasan I, Junejo AM, Dawani ML. Etiology and Outcome of Acute Renal Failure in Pregnancy. JCPSP 2009;19(11):714-7

14. Chaudhri N, But GUD, Masroor I, Qureshi MA, Shehzad MN, Abbasi MSR, Karmani JK. Spectrum and Short Term Outcome of Pregnancy related Acute Renal Failure among Women admitted in Nephrology Ward; Pakistan Institute of Medical Sciences. Ann Pak. Inst. Med. Sci. 2011;7(2):57-61

15. Novotny S, Lee-Plenty N, Wallace K, Kassahun-Yimer W, Jayaram A, Bofill J, Martin JN. Acute kidney injury associated with preeclampsia or hemolysis, elevated liver enzymes and low platelet syndrome. Pregnancy Hypertens. 2020; 19:94 - 9.

16. Naqvi R. Hemolytic uremic syndrome associated with pregnancy: outcome from acute kidney injury. Pak J Med Sci. 2020;36(6):1153-7.

17. Slemmons J, Bogert L. The uric acid content of maternal and fetal blood. J Biol Chem. 1917;32:63-9

18. Kang DH, Finch J, Nakagawa T, Karumanchi SA, Kanellis JG, Johnson RJ. Uric acid, endothelial dysfunction and pre-eclampsia: Searching for a pathogenetic link. J Hypertens.2004;22(2):229-35.

19. Vikse BE, Irgens LM, Leivestad T, Skjaerven R, Iversen B.M. Preeclampsia and the risk of end-stage renal disease. N Engl J Med. 2008; 359:800 - 9.

20. Ye W, Shu H, Yu Y, Li H, Chen L, Liu J, et al. Acute kidney injury in patients with HELLP syndrome. Int Urol Nephrol. 2019; 51:1199 - 206.

21. Garovic VD. The Role of the Podocyte in Preeclampsia. Clin J Am Soc Nephrol. 2014;9(8):1337-40

22. Martin JN J, Brewer J, Wallace K, Sunesara I, Canizaro A, Blake P, et al. HELLP syndrome and composite major maternal morbididty:Importance of Mississippi classification system. J Matern Fetal and Neonatal Med. 2013; $26: 1201-6$ 


\section{AKI \& Preeclampsia}

23. Khattak MI, Sabir S, Khattak SN, Tahir N. Spectrum of Acute Kidney Injury (AKI) in Pregnancy. Journal of Rawalpindi Medical College (JRMC); 2014;18(2):267-269

24. Brown MA, Magee LA, Kenny LC, Karumanchi SA, McCarthy FP, Saito S, Hall DR, Warren CE, Adoyi G, Ishaku S. The hypertensive disorders of pregnancy: ISSHP classification, diagnosis \& management recommendations for international practice. Pregnancy Hypertens. 2018;23:24 - 43.

25. Linsell L, Malouf R, Morris J, Kurinczuk JJ, Marlow N. Risk Factor Models for Neurodevelopmental Outcomes in Children Born Very Preterm or With Very Low Birth Weight: A Systematic Review of Methodology and Reporting. Am. J. Epidemiol. 2017;185:601 - 12.

26. Vaught AJ, Kovell LC, Szymanski LM, Mayer SA, Seifert SM, Vaidya D, Murphy JD, Argani C, O'Kelly A, York S, Ouyang P, Mukherjee M, Zakaria S. Acute Cardiac Effects of Severe Pre-Eclampsia. J Am Coll Cardiol.2018;72(1):1-11

27. Naqvi R. Epidemiological trends in community acquired acute Kidney Injury in Pakistan: 25 years Experience from a Tertiary Care Renal Unit. Pak J Med Sci. 2021;37(2):312-19 .doi:https://doi.org/10.12669/pjms.37.2.3876 